\title{
Scheduling in Dense Small Cells With Successive Interference Cancellation
}

\author{
Ronghui Hou, Yarong Xie, King-Shan Lui, and Jiandong Li
}

\begin{abstract}
Smart interference management has been receiving attention to improve network throughput. Successive interference cancellation (SIC) is one of the promising techniques, which allows multiple concurrent transmissions from different transmitters to the same receiver. We study the scheduling issue with SIC in dense small cells. This paper proposes a novel scheduling framework, which facilitates us to develop practical algorithms to find the solution.
\end{abstract}

Index Terms-Scheduling, successive interference cancellation, small cells.

\section{INTRODUCTION}

$\mathbf{I}$ $\mathrm{T}$ is well known that managing interference intelligently is an efficient way to improve the throughput in wireless networks [1]. Successive interference cancellation (SIC) is a promising technique to mitigate interference when the signals overlap [1]. Suppose a certain node receives two signals of power levels $P_{1}$ and $P_{2}$, where $P_{1}<P_{2}$. If $P_{2} /\left(P_{1}+\delta^{2}\right) \geq \beta$ and $P_{1} / \delta^{2} \geq \beta$, where $\delta^{2}$ is the power level of the ambient noise and $\beta$ is a certain value. By using SIC, the node can first decode the signal with power level $P_{1}$ and then decode the signal with power level $P_{2}$. Theoretically, SIC can allow more than two concurrent transmissions meant to the same receiver.

This paper studies how to schedule transmissions with SIC when several mobile stations want to transmit to small-cell base stations (SBS). Following [2], there is a small cell gateway (SGN) in the network, and the SGN possesses state information of the whole network to make the scheduling decision. We first use an example to illustrate how scheduling affects throughput. In Fig. 1, there are three small-cell base stations (SBS1, SBS2, and SBS3) and six mobile stations $\left(m_{1}\right.$ to $\left.m_{6}\right)$. Let $P_{i, j}$ denote the power level of the signal from node $i$ that is received by node $j$. The received power levels at SBS1 are assumed as: $P_{1,1}>P_{2,1}>$ $P_{3,1}, P_{1,1} /\left(P_{2,1}+P_{3,1}+\delta^{2}\right) \geq \beta, P_{2,1} /\left(P_{3,1}+\delta^{2}\right) \geq \beta$, but

Manuscript received January 6, 2014; accepted April 13, 2014. Date of publication April 22, 2014; date of current version June 6, 2014. This work was supported in part by the National Natural Science Foundation of China (Grants Nos. 61231008 and 61101143), the important national science \& technology specific projects 2013ZX03004007-003, the HKU small project fund (104001905), the Fundamental Research Funds for the Central Universities K50511010006, and the 111 Project under Grant B08038 The associate editor coordinating the review of this paper and approving it for publication was I. Guvenc.

R. Hou, Y. Xie and J. Li are with the State Key Lab of Integrated Service Networks, Xidian University, Xi'an 710071, China (e-mail: rhhou@ xidian.edu.cn; jdli@mail.xidian.edu.cn).

K.-S. Lui is with the Department of Electrical and Electronic Engineering, The University of Hong Kong, Pokfulam, Hong Kong (e-mail: kslui@ eee.hku.hk).

Color versions of one or more of the figures in this paper are available online at http://ieeexplore.ieee.org.

Digital Object Identifier 10.1109/LCOMM.2014.2318310

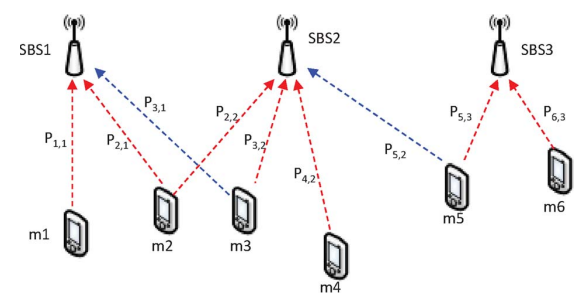

Fig. 1. Illustration for scheduling with SIC.

$P_{3,1} / \delta^{2}<\beta$. This implies that SBS1 can successfully decode the signals from $m_{1}$ and $m_{2}$ when $m_{1}, m_{2}$, and $m_{3}$ transmit concurrently. The received power levels at SBS2 are assumed as: $P_{2,2}>P_{3,2}>P_{4,2}>P_{5,2}, P_{2,2} /\left(P_{3,2}+P_{4,2}+\delta^{2}\right) \geq \beta$, $P_{3,2} /\left(P_{4,2}+P_{5,2}+\delta^{2}\right) \geq \beta, P_{4,2} /\left(P_{5,2}+\delta^{2}\right) \geq \beta$, but $P_{2,2} /$ $\left(P_{3,2}+P_{4,2}+P_{5,2}+\delta^{2}\right)<\beta$ and $P_{5,2} / \delta^{2}<\beta$. Note that $P_{2,2} /\left(P_{3,2}+P_{4,2}+\delta^{2}\right) \geq \beta$ implies that $P_{2,2} /\left(P_{4,2}+P_{5,2}+\right.$ $\left.\delta^{2}\right) \geq \beta$ and $P_{2,2} /\left(P_{3,2}+P_{5,2}+\delta^{2}\right) \geq \beta$. Then, SBS2 cannot correctly decode any signal if all four mobile stations $\left(m_{2}\right.$ to $m_{5}$ ) transmit concurrently. It can decode some signals if only three of the four transmit at the same time. The received power levels at SBS3 are assumed as: $P_{5,3}>P_{6,3}$, $P_{5,3} /\left(P_{6,3}+\delta^{2}\right)<\beta, P_{5,3} / \delta^{2} \geq \beta$, and $P_{6,3} / \delta^{2} \geq \beta$. SBS3 cannot decode any signal when both $m_{5}$ and $m_{6}$ transmit concurrently. Therefore, if we let $m_{1}, m_{2}, m_{4}$, and $m_{5}$ transmit concurrently, $m_{3}$ and $m_{6}$ should not transmit to avoid conflict at SBS2 and SBS3, respectively. On the other hand, if we keep $m_{5}$ to be idle, all the other stations can transmit concurrently. The number of concurrent transmissions in the former schedule is four, while that of the latter one is five.

Some existing works on scheduling with SIC can be found. [3] studies the uplink scheduling problem with SIC in a network with a set of stations and a single base station. The solution in [3] is not suitable in our situation where a network has multiple base stations. [1] and [4] theoretically formulate the scheduling problem with SIC in multi-hop wireless networks as a mixedinteger linear programming. The computational overhead to find a solution is huge. The works in [5]-[7] propose using the simultaneity graph to capture the effect of SIC. However, it is difficult to identify a conflict-free scheduling solution based on the simultaneity graph. Therefore, we propose a new graph model to formulate the scheduling problem with SIC in small cell networks. By using our constructed graph, an optimal schedule can be obtained by identifying a maximal weighted independent set.

As mentioned in [3], different scheduling problems may aim at different objectives. Two commonly studied objectives are maximizing throughput and enhancing fairness among mobile stations. This paper focuses on throughput maximization. We will also discuss how to extend our method to other scheduling objectives. 


\section{Proposed Scheduling Framework}

\section{A. Network Model}

We consider the network composed by a set of small-cell base stations $(\mathrm{SBS}), \mathcal{S}$, and a set of mobile stations, $\mathcal{M}$. Following [1], the transmitting power at each mobile station is fixed. We do not consider link rate adaptation in our model and assume that the data rate for each successful transmission is $R=B \log (1+\beta)$, where $B$ is the channel bandwidth. We consider the uplink transmissions from mobile stations to SBSs. An SBS can decode the signal from a mobile station only when the signal is strong enough. Given mobile station $j$ and SBS $i$, if $P_{j, i} / \delta^{2} \geq \beta$, it means that SBS $i$ can successfully decode the signal from $j$ if there is no interference from other stations, and we call $j$ is in the decoding range of $i$. A signal that is not strong enough for an SBS to decode can be strong enough for the SBS to hear. Given $\beta^{\prime}<\beta$, when $\beta^{\prime} \leq\left(P_{j, i} / \delta^{2}\right)<\beta$, $i$ can hear the signal from $j$ but cannot decode. In this case, we call $j$ is in the hearing range of $i$. When $\left(P_{j, i} / \delta^{2}\right)<\beta^{\prime}$, the signal from $j$ does not affect the reception at node $i$.

In practice, each mobile station can first increase its transmitting power, so that each small-cell base station can then estimate the path loss of each mobile station it hears from, and then determine the mobile stations located within the decoding range and the hearing range. Denote by $\mathcal{M}\left(s_{i}\right)$ the mobile stations that SBS $s_{i}$ can hear or decode the signals from. We assume that the mobile stations transmit with the same frequency band. A mobile station can be heard by multiple SBSs, but it requires only one of them to decode the signal successfully. This work aims at finding a schedule such that the number of concurrent decodable transmissions from mobile stations is maximized.

\section{B. SIC Opportunities}

Suppose that SBS $s_{i}$ receives $J$ signals from the stations in $\mathcal{M}\left(s_{i}\right)$. For simplicity, we call these stations $1,2, \ldots, J$. Without loss of generality, let the received power levels of the signals satisfy $P_{1, i} \leq P_{2, i} \leq \cdots \leq P_{J, i}$. To apply SIC, $s_{i}$ should decode the signals in the order of $J, J-1, \ldots, 1$. The signal with received power $P_{j, i}$ can be decoded successfully if and only if

$$
\begin{aligned}
& \frac{P_{J, i}}{\sum_{k=1}^{J-1} P_{k, i}+\delta^{2}} \geq \beta \\
& \frac{P_{J-1, i}}{\sum_{k=1}^{J-2} P_{k, i}+\delta^{2}} \geq \beta \\
& \vdots \quad \\
& \frac{P_{j, i}}{\sum_{k=1}^{j-1} P_{k, i}+\delta^{2}} \geq \beta .
\end{aligned}
$$

Definition 1-[SIC Opportunity]: $(M, s), M \in \mathcal{M}\left(s_{i}\right)$, is an SIC opportunity if when the stations in $M$ transmit concurrently, $s$ can decode all the signals from the stations in $M$ that are in its decoding range.

$J$ mobile stations can form an SIC opportunity corresponding to $s_{i}$ when either one of the following is satisfied:

1) Each of the $J$ signals can be decoded.

2) $\left(P_{j-1, i} / \delta^{2}\right)<\beta$, and the signals from stations $j$ to $J$ can be decoded.

When $\left(P_{j-1, i} / \delta^{2}\right)<\beta$ (second case), stations 1 to $j-1$ are not in the decoding range of $s_{i}$. Nevertheless, $s_{i}$ can still decode

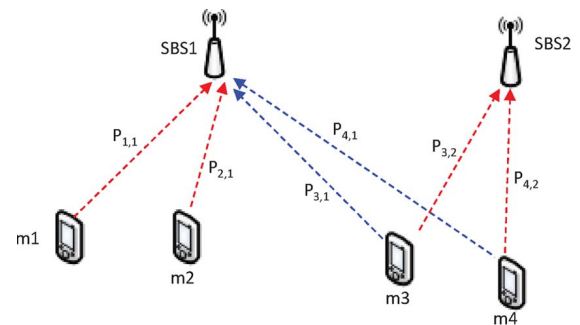

Fig. 2. Illustration for SIC opportunities.

the signals from stations $j$ to $J$. From the perspective of $s_{i}$, all the $J$ stations can transmit concurrently and is thus an SIC opportunity to $s_{i}$.

On the other hand, if $\left(P_{j-1, i} / \delta^{2}\right)>\beta$, but the signal from station $j-1$ cannot be decoded due to the interference from the other stations from 1 to $j-2$, there is no SIC opportunity formed by the $J$ stations. Given SBS $s_{i}$, there are at most $\left|\mathcal{M}\left(s_{i}\right)\right|^{K}$ SIC opportunities, where $K$ is the maximum number of concurrent transmissions by using SIC. In practice, we can limit $K$ to reduce the computational overhead.

In Fig. 2, where only those transmissions within the hearing and decoding ranges are shown as dotted arrows, assume $P_{1,1}>P_{2,1}>P_{3,1}>P_{4,1}, P_{1,1} /\left(P_{2,1}+P_{3,1}+P_{4,1}+\delta^{2}\right) \geq$ $\left.\beta, P_{2,1} / P_{3,1}+P_{4,1}+\delta^{2}\right) \geq \beta$, and $\left(P_{3,1} / \delta^{2}\right)<\beta$. According to Definition $1,\left(\left\{m_{1}, m_{2}, m_{3}, m_{4}\right\}, 1\right)$ is an SIC opportunity. When the four stations transmit concurrently, SBS1 can successfully decode the signals from $m_{1}$ and $m_{2}$. We further assume that SBS2 can decode the signal when either $m_{3}$ or $m_{4}$, but not both, is transmitting. Then, the optimal schedule for this example should be $\left\{m_{1}, m_{2}, m_{3}\right\}$ or $\left\{m_{1}, m_{2}, m_{4}\right\}$, but not all four stations transmit concurrently. We are going to develop an appropriate graph model based on the SIC opportunities, so as to facilitate us to identify optimal scheduling.

\section{Concurrent Transmission Graph and Scheduling}

In this section, we propose a graph model, called Concurrent Transmission Graph $(C G)$, to reflect the conflict among the different transmissions. Each vertex in the CG corresponds to an SIC opportunity. If the network contains $S$ SBSs, and each SBS has $Z$ SIC opportunities. There are totally $S Z$ vertices in the corresponding CG. Two vertices are connected by an edge if the corresponding SIC opportunities conflict with each other. Two SIC opportunities are in conflict when both opportunities cannot co-exist, and we detect whether two SIC opportunities conflict with each other based on the following:

Property 1: SIC opportunities $\left(M_{1}, s\right)$ and $\left(M_{2}, s\right)$ conflict with each other.

As each SBS should select a single subset of stations to transmit concurrently, two SIC opportunities with the same SBS should not co-exist in a schedule.

Property 2: Given two SIC opportunities $\left(M_{1}, s_{1}\right)$ and $\left(M_{2}, s_{2}\right)$, where $s_{1} \neq s_{2}$, let $M_{1}^{\prime}$ (or $M_{2}^{\prime}$ ) be the set of stations in $M_{2}$ (or $M_{1}$ ) that are in the decoding or hearing range of $s_{1}$ (or $s_{2}$ ). If $M_{1}^{\prime} \subseteq M_{1}$ and $M_{2}^{\prime} \subseteq M_{2},\left(M_{1}, s_{1}\right)$ and $\left(M_{2}, s_{2}\right)$ do not conflict with each other; they conflict otherwise.

$\left(M_{1}, s_{1}\right)$ is an SIC opportunity because $s_{1}$ can decode those signals from stations $M_{1}$ that are within its decoding range. $M_{1}^{\prime} \subseteq M_{1}$ implies that the stations in $\mathcal{M}\left(s_{1}\right)$ that are 


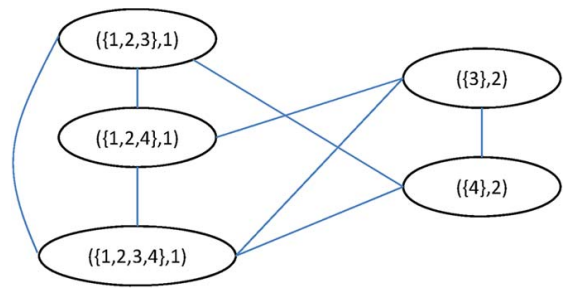

Fig. 3. Illustration for CG.

transmitting under SIC opportunities $\left(M_{2}, s_{2}\right)$ are already included in $M_{1}$. Therefore, $\left(M_{2}, s_{2}\right)$ does not interfere the decoding of $s_{1}$. Similarly, when $M_{2}^{\prime} \subseteq M_{2},\left(M_{1}, s_{1}\right)$ still allows $s_{2}$ to decode the same signals. However, when $M_{1}^{\prime} \nsubseteq \subseteq M_{1}$, it means there exists $m \in M_{1}^{\prime}$ but $m \notin M_{1} . m$ may interfere the decoding at $s_{1}$, and we assume there is a conflict. Note that if $m$ does not interfere the decoding at $s_{1},\left(M_{1} \cup\{m\}, s_{1}\right)$ must be an SIC opportunity. Therefore, it is fine to assume a conflict between $\left(M_{1}, s_{1}\right)$ and $\left(M_{2}, s_{2}\right)$.

We now present an example. Fig. 3 shows part of the CG corresponding to Fig. 2. As we mentioned earlier, $(\{1,2,3,4\}$, $1),(\{1,2,3\}, 1)$, and $(\{1,2,4\}, 1)$ are all SIC opportunities. Given $S_{1}=(\{1,2,3\}, 1)$ and $S_{2}=(\{3\}, 2), S_{1}$ contains all the stations in $S_{2}$, and $S_{2}$ also contains all the stations in $S_{1}$ that SBS2 can hear from. Thus, both SIC opportunities do not conflict with each other. Given $S_{3}=(\{1,2,3\}, 1)$ and $S_{4}=(\{4\}, 2)$, since $S_{4}$ does not contain station 3 that SBS2 can hear from, the SIC opportunities conflict with each other. In CG, each independent set represents a conflict-free transmission schedule. Our scheduling problem is thus reduced to find an appropriate independent set in CG. For instance, $\{(\{1,2,3\}, 1),(\{3\}, 2)\},\{(\{1,2,4\}, 1),(\{4\}, 2)\}$, and $\{(\{1$, $2,3,4\}, 1)\}$ are all independent sets. However, three mobile stations, $\left\{m_{1}, m_{2}, m_{3}\right\}$ or $\left\{m_{1}, m_{2}, m_{4}\right\}$ by using the first two scheduling decisions are served, while only two stations are served in the last one. Therefore, we should define a weight for the independent set to identify the one that facilitates that most stations to transmit and be decoded.

Definition 2: The weight of an SIC opportunity $(M, s)$, $\omega(M, s)$, is defined as the number of stations that $s$ can correctly decode their signals.

Definition 3: The weight of an independent set $\mathcal{I}=$ $\left\{\left(M_{1}, s_{1}\right), \ldots,\left(M_{|\mathcal{I}|}, s_{|\mathcal{I}|}\right)\right\}$ is defined as the total number of stations from which at least one SBS in $\left\{s_{1}, \ldots, s_{|\mathcal{I}|}\right\}$ can correctly decode the signal.

By Definition 3, our problem is reduced to the problem of finding an independent set with the maximum weight. Since finding all the independent sets is computationally expensive, we propose a greedy algorithm (Algorithm 1) to identify an independent set. In our algorithm, we first select the vertex $(M, s)$ in the CG with the maximum weight. We then remove all the vertices which are connected with $(M, s)$, since these SIC opportunities conflict with the selected SIC opportunity. Let $\bar{M}$ denote the set of stations whose signals can be correctly decoded. After selecting a vertex, we update the weight of each remaining vertex by only considering the stations not contained in $\bar{M}$. Note that although we update the weight of each vertex, the CG constructed initially should not be changed. Afterwards, we continue to select the next vertex with the maximum weight. This procedure continues untill no vertex is contained in the CG. Since any two of the selected vertices do not conflict with each other, the mobile stations specified by the selected vertices can transmit concurrently.

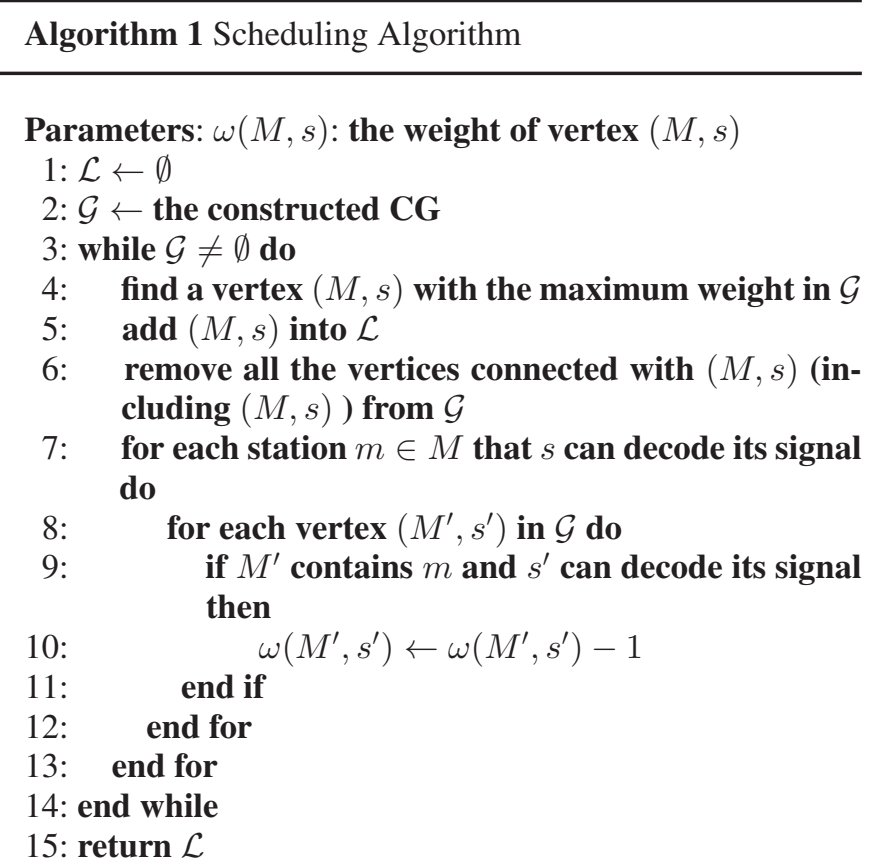

Our method can be extended to consider other scheduling objectives. For instance, if we want to schedule all stations with the minimum number of time slots, we can first use Algorithm 1 to identify the set of concurrent transmitting stations. Afterwards, we remove all the selected stations from the network and reconstruct the concurrent transmission graph. Algorithm 1 is used again to identify another set of stations to transmit concurrently. This process terminates when all the stations are scheduled.

\section{NUMERICAL EXPERIMENTS}

We conducted simulations to evaluate the performance of our proposed scheduling methods. In this paper, we reduce the scheduling problem with SIC to the problem of identifying an independent set. We tested two methods to find independent sets: one is the exhaustive method for identifying all the independent sets so as to obtain the optimal solution, which is computationally expensive; another one is the proposed greedy algorithm to identify an independent set, as shown in Algorithm 1. We compared our algorithms with the optimal scheduling solution in [1] obtained by solving a mixed-integer linear programming.

We randomly generate several small cells in a square region of $250 * 250$. The mobile stations are also randomly deployed in the region. We normalize all units for distance, data rate, and power with appropriate dimensions. Following [1], the transmission power of each node is set to $P=1$. For simplicity, we assume that channel gain $g_{i, j}$ only includes the path loss between station $i$ and SBS $j$, and is gained by $d_{i, j}^{\gamma}$, where $d_{i, j}$ denotes the distance and $\gamma=3$. The power of ambient noise is $\delta^{2}=10^{-6}$. We set $\beta=1$.

We tried five different network instances, and the simulation results are the average on five results. Fig. 4(a) and (b) show 


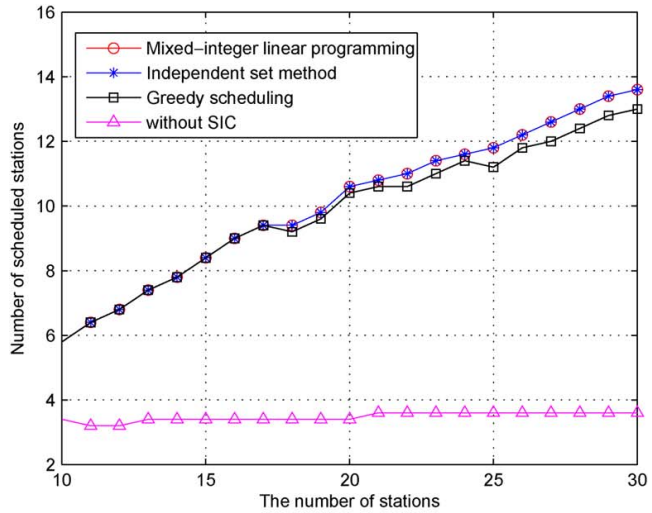

(a)

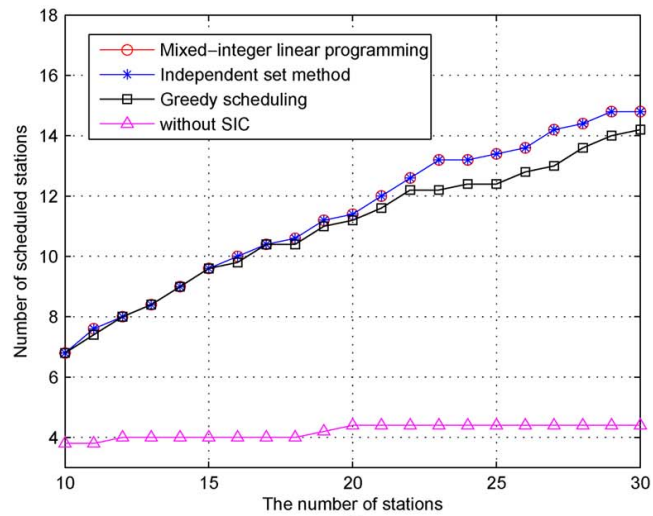

(b)

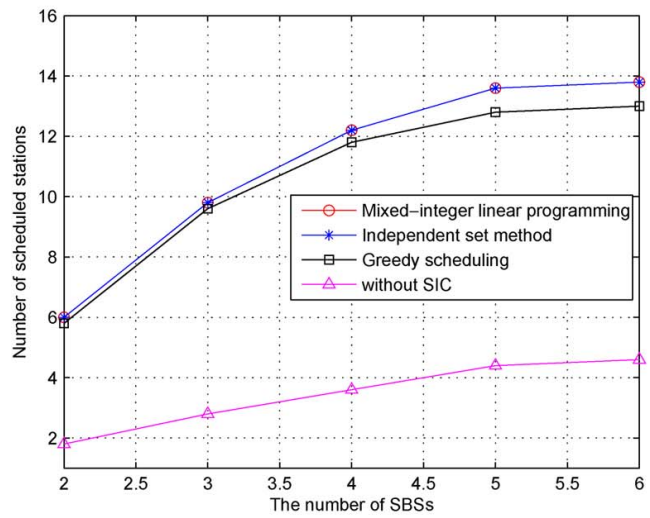

(c)

Fig. 4. Simulation results. (a) Scenario with 4 SBSs. (b) Scenario with 5 SBSs. (c) Performance vs number of SBSs.

the simulation results when the number of SBSs are 4 and 5, respectively. We observe that our graph-based method produces the same results as the mixed-integer linear programming when the optimal independent set is identified. This verifies that our constructed graph-based method can correctly model the problem, and the optimization problem is equivalent to the problem of finding all independent sets. As the number of SBSs increases, more stations can be scheduled. Without SIC, at most one station can be served by a SBS, and so at most four (or five) stations are scheduled in Fig. 4(a) (or Fig. 4(b)). The simulation results show that SIC can effectively improve network throughput. During the simulations, we find that the independent set maintaining the maximum number of stations may not be the maximal independent set (the independent set that contains the maximum number of vertices in the $\mathrm{CG}$ ).
It is possible that all vertices in a maximal independent set contains the same stations, such that the weight of this set may not be the maximum. It implies that existing algorithms on finding the maximal independent set may not work well in our problem. Since finding the optimal solution by identifying all the independent sets is computationally expensive, we propose a greedy method to identify an independent set. The simulation results show that the greedy method schedules one less station than the optimal solution on average.

To understand the benefits of SIC, we study how many mobile stations can be supported when number of SBSs varies. We put 26 mobile stations in the network and changes the number of SBSs from 2 to 6 . The number of stations supported can be observed in Fig. 4(c). As the number of SBSs increases, the number of scheduled stations increases. The throughput no longer improves when the number of SBSs is over a certain threshold. When the ratio of interference to signal is high enough, SIC first decodes the interference signal, and then decodes the desired signal after removing the interference signal. When the number of SBSs is larger than a threshold, the ratio of interference to signal would not change a lot, and so the number of SIC opportunities would not increase significantly. Thus, the network throughput would not change when there are more SBSs.

Generally speaking, SIC can efficiently improve the network throughput with intelligent scheduling scheme. Our graphbased method can correctly model the scheduling problem with SIC. The proposed greedy method works well to select the stations to be scheduled; moreover, the greedy method is simple and can quickly develop a solution, which is practical in the small cell network.

\section{CONCLUSION}

This paper studied the problem of scheduling with SIC in dense small cell networks, which is reduced to the problem of finding all the independent sets by using the proposed graph-based method. We also proposed a fast algorithm to find a feasible schedule. Our simulation results demonstrated the performance of our lgorithm. We did not consider the effect of SINR on transmission rate in this paper. We plan to explore it in the future.

\section{REFERENCES}

[1] C. Jiang et al., "Squeezing the most out of interference: An optimization framework for joint interference exploitation and avoidance," in Proc. IEEE INFOCOM, Apr. 2012, pp. 424-432.

[2] W. Ni, I. B. Collings, and R. Liu, "Decentralized user-centric scheduling with low rate feedback for mobile small cells," IEEE Trans. Wireless Commun., vol. 12, no. 12, pp. 6106-6120, Dec. 2013.

[3] M. Ghaderi and M. Mollanoori, "Uplink scheduling in wireless networks with successive interference cancellation," IEEE Trans. Mobile Comput., to be published.

[4] L. Shi, Y. Shi, Y. Ye, Z. Wei, and J. Han, "An efficient interference management framework for multi-hop wireless networks," in Proc. IEEE WCNC, 2013, pp. 1434-1439.

[5] S. Lv, W. Zhuang, X. Wang, and X. Zhou, "Scheduling in wireless Ad Hoc networks with successive interference cancellation," in Proc. IEEE INFOCOM, Apr. 2011, pp. 1-5.

[6] S. Lv, W. Zhuang, X. Wang, C. Liu, and X. Zhou, "Maximizing capacity in the sinr model in wireless networks with successive interference cancellation," in Proc. IEEE ICC, 2011, pp. 1-6.

[7] S. Lv, W. Zhuang, X. Wang, and X. Zhou, "Context-aware scheduling in wireless networks with successive interference cancellation," in Proc. IEEE ICC, 2011, pp. 1-5. 